\title{
Preface
}

\section{Normal Electrophysiology, \\ Substrates, and the Electrocardiographic Diagnosis of Cardiac Arrhythmias}

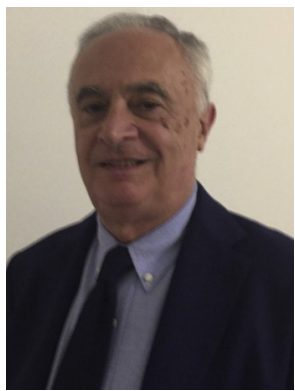

Luigi Padeletti, MD

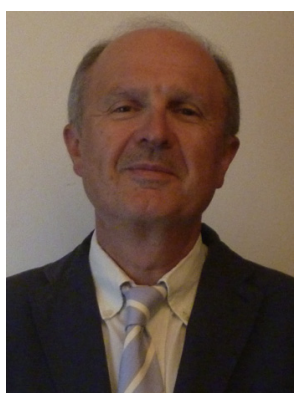

Giuseppe Bagliani, MD

Editors

The purpose of this and the next issue of Cardiac Electrophysiology Clinics is to treat in depth and to review extensively any kind of cardiac arrhythmia defining the correct diagnostic approach on the surface electrocardiogram.

In the last decades, progress in basic research and in clinical electrophysiology has continuously increased our knowledge of the mechanisms underlying cardiac arrhythmias, furnishing evidence of previously undetected abnormalities of the formation and conduction of the cardiac impulse that help to make a correct electrocardiographic diagnosis of complex arrhythmias.

Basically, the focus of the issue is the surface electrocardiogram, with all the information we can obtain (from the cell electrophysiology to the neural control) in normal conditions and during rhythm disturbances. As a consequence, we followed a learning process starting from the description of the single electrocardiographic waves and intervals, which provides insight into the physiologic and pathologic cause, the consequent morphologies, and the criteria of normality and abnormality. Successively, we developed the articles addressing the single arrhythmias originating in each cardiac structure, following a rationally based analytic approach to the accurate diagnosis of arrhythmias.

Our love for arrhythmology was aroused many years ago, when we were young fellows, by the papers and books of Katz, Pick, Langendorf, and Schamroth, Their methodology and rationality remain unreachable. Paraphrasing Isaac Newton, if next generations of arrhythmologists have seen further, it is by standing on the shoulders of these giants.

As the editors of this issue of Cardiac Electrophysiology Clinics, we would like to express our gratitude to the coauthors of the individual articles for their dedication to the production of these two issues. The donation of their expertise, time, and effort was essential. This issue also would not 
have been possible without the dedicated assistance of Donald Mumford. The support and encouragement of Ranjan Thakur and Andrea Natale were also essential.

Luigi Padeletti, MD Heart and Vessels Department University of Florence Largo Brambilla, 3 50134 Florence, Italy

IRCCS Multimedica Cardiology Department Via Milanese, 300 20099 Sesto San Giovanni, Italy
Giuseppe Bagliani, MD Arrhythmology Unit Cardiology Department Foligno General Hospital Via Massimo Arcamone 06034 Foligno (PG), Italy

Cardiovascular Diseases Department University of Perugia Piazza Menghini 1 06129 Perugia, Italy

E-mail addresses: luigi.padeletti@unifi.it (L. Padeletti) giuseppe.bagliani@tim.it (G. Bagliani) 